\title{
ALÉM DA FILOSOFIA ALTRUÍSTA: UM ESTUDO SOBRE OS DETERMINANTES DO ENVOLVIMENTO DO CONSUMIDOR DE ALIMENTOS ORGÂNICOS
}

\author{
BEYOND ALTRUISTIC PHILOSOPHY: A STUDY ON THE \\ DETERMINANTS OF ORGANIC FOOD CONSUMER \\ INVOLVEMENT
}

\section{MÁS ALLÁ DE LA FILOSOFÍA ALTRUÍSTA: UN ESTUDIO SOBRE LOS DETERMINANTES DEL ENVOLVIMIENTO DEL CONSUMIDOR DE ALIMENTOS ORGÁNICOS}

Artigo aprovado em fast-track do XXI SEMEAD

José Eduardo de Melo Barros

Mestre em Administração e Desenvolvimento

Rural - Universidade Federal Rural de

Pernambuco, Recife, Brasil

Professor no Curso de Administração - Centro Universitário Uninabuco, Paulista, Brasil

jeduardobarros@hotmail.com

\section{Romilson Marques Cabral}

Doutor em Administração - Universidade

Federal da Bahia, Salvador, Brasil

Professor adjunto no Departamento de

Administração - Universidade Federal Rural de

Pernambuco, Recife, Brasil

romilsonmarquescabral10@gmail.com

\section{Brigitte Renata Bezerra de Oliveira}

Doutora em Administração - Universidade

Federal de Pernambuco, Recife, Brasil

Professora adjunta no Departamento de

Administração - Universidade Federal Rural de Pernambuco, Recife, Brasil

renataoliveira@ufrpe.br

\section{Francisco Vicente Sales de Melo}

Doutor em Administração - Universidade

Federal de Pernambuco, Recife, Brasil

Professor adjunto no Departamento de Ciências

Administrativas - Universidade Federal de

Pernambuco, Recife, Brasil

vicsmelo@gmail.com

\section{Tarcísio Regis de Souza Bastos}

Mestre em Administração e Desenvolvimento

Rural - Universidade Federal Rural de

Pernambuco, Recife, Brasil

Doutorando em Administração - Universidade

Federal de Pernambuco, Recife, Brasil

tarcisioregis1@hotmail.com
Contextus

ISSN 1678-2089

ISSNe 2178-9258

Organização: Comitê Científico Interinstitucional Editor Científico: Diego de Queiroz Machado Editor Executivo: Carlos Daniel Andrade Avaliação: double blind review pelo SEER/OJS

Recebido em 15/10/2018

Aceito em 15/10/2018

$2^{a}$ versão aceita em 29/10/2018 


\title{
RESUMO
}

A consciência de preservação ecológica e a busca por alimentação saudável foram ideias disseminadas na década de 1970. No final da seguinte, novos parâmetros se somaram à motivação ideológica. Este artigo teve por objetivo compreender o envolvimento dos consumidores de produtos orgânicos. A revisão de literatura desenvolvida neste trabalho aborda o construto de envolvimento, sua multidimensionalidade e sua mensuração, bem como os determinantes do alto envolvimento com orgânicos. Os dados foram coletados por meio de survey on-line, cuja amostra se compunha de 412 respondentes válidos. Utilizaram-se técnicas de estatística descritiva e inferencial, sobretudo análise fatorial e regressão logística. De modo geral, é possível afirmar que as hipóteses foram parcialmente confirmadas. Conclui-se que o envolvimento com orgânicos se dá de maneira multifacetada e os determinantes que distinguem os indivíduos altamente envolvidos podem ser associados a circunstâncias egoístas e a situações de compra específicas, como maior frequência e disponibilidade em pagar preços superiores.

Palavras-chave: alimentos orgânicos; consumidor de orgânicos; motivação de consumo; envolvimento; determinantes do envolvimento.

\begin{abstract}
The awareness of ecological preservation and the search for healthy food were widespread ideas in the 1970s. In the late 1980s, new parameters were added to that ideological motivation. This article aimed to understand the involvement of organic product consumers. The literature review developed in this work approaches the involvement construct, its multidimensionality and its measurement, as well as the determinants of high involvement with organic food. The data were collected through an online survey, whose sample consisted of 412 valid respondents. Descriptive and inferential statistics techniques, mainly factorial analysis and logistic regression, were used. In general, it is possible to affirm that the hypotheses were partially confirmed. It is concluded that involvement with organic products occurs in a multifaceted way and the determinants that distinguish highly involved individuals may be associated with selfish circumstances and specific buying situations, such as greater regularity and willingness to pay higher prices.
\end{abstract}

Keywords: Organic food; Organic consumer; Consumer motivation; Involvement; Determinants of involvement.

\section{RESUMEN}

La conciencia de preservación ecológica y la búsqueda de alimentación sana fueron ideas diseminadas en la década de 1970. Al final de la siguiente, nuevos parámetros se sumaron a la motivación ideológica. Este artículo tuvo por objetivo comprender el envolvimiento de los consumidores de productos orgánicos. La revisión de literatura desarrollada en este trabajo aborda el constructo de envolvimiento, su multidimensionalidad y su medición, así como los determinantes del alto envolvimiento con orgánicos. Los datos fueron recolectados por medio de survey on-line, cuya muestra se componía de 412 respondedores válidos. Se utilizaron técnicas de estadística descriptiva e inferencial, sobre todo análisis factorial y regresión logística. En general, es posible afirmar que las hipótesis se han confirmado parcialmente. Se concluye que el envolvimiento con orgánicos se da de manera multifacética y los determinantes que distinguen a los individuos altamente envueltos pueden ser asociados a circunstancias egoístas y a situaciones de compra específicas, como mayor regularidad y disponibilidad en pagar precios superiores.

Palabras clave: alimentos orgánicos; consumidor de orgánicos; motivación de consumo; envolvimiento; determinantes del envolvimiento.

\section{INTRODUÇÃO}

Acompanhando o movimento mundial na década de 1970, o Brasil desenvolveu sua produção orgânica fundamentando-se, essencialmente, nos movimentos filosóficos que preconizavam o retorno do contato com a terra como alternativa à ordem consumista da sociedade moderna. A vertente ecológica desse movimento promoveu a recusa da chamada agricultura moderna, ao estabelecer uma lógica de comercialização dos produtos em que a relação entre produtor e consumidor era direta, de modo semelhante a uma ação coletiva "entre 
amigos" (ORMOND et al., 2002). Esses autores explicam que a consciência de preservação ecológica e a busca por alimentação saudável foram ideias disseminadas na década de 1980, quando tiveram início e se ampliaram diversas cooperativas de produção e consumo de produtos naturais, como também restaurantes especializados nesse tipo de alimentação.

Em contrapartida, Dalcin et al. (2014) afirmam que, no final da década de 1980, os parâmetros de oferta e demanda extrapolaram a motivação ideológica. Conforme explicam, a razão mais relevante para a ampliação do mercado foi a mudança nas preferências do consumidor brasileiro, pois aumentou consideravelmente o número de indivíduos que consideram o valor nutricional e a qualidade dos alimentos que consomem. Nessa fase, os produtos orgânicos passaram também a ser distribuídos em cadeias de suprimento mais longas, expandindo-se para as grandes redes de varejo brasileiras (GUIVANT, 2003).

O consumo de alimentos orgânicos provém de uma ideologia relacionada a um conjunto de valores particulares, que influenciam atitudes e comportamentos de consumo (SCHIFFERSTEIN; OPHUIS, 1998). Tais valores incluem altruísmo (relação com os outros), ecologia (harmonia com um universo sustentável), universalismo (bem-estar do homem com a natureza), benevolência (bem-estar entre as pessoas próximas), espiritualidade (harmonia interna e unidade com a natureza) e autodireção (pensamento e ação independentes) (SCHIFFERSTEIN; OPHUIS, 1998; HUGHNER et al., 2007). Em resumo, a literatura aponta razões ambientais, sociais, econômicas e éticas para o consumo de alimentos orgânicos (ZANOLI; NASPETTI, 2002; HUGHNER et al., 2007; AERTSENS et al., 2009; TENG; LU, 2016). Dalcin et al. (2014) já relataram a existência de um perfil típico de consumidor, com motivação conceitual decorrente de uma ideologia ecológica e social altruísta, e outro perfil de indivíduos motivados por tendências de mercado relativas à saúde e à nutrição, sobre os quais o apelo orgânico possui conotação mais individualizada.

Em estudos sobre o comportamento do consumidor e a tomada de decisão, o envolvimento é um construto cujas motivações são antecedentes. O envolvimento reflete o nível de motivação do indivíduo para processar informações sobre um produto ou serviço que o auxiliará a solucionar um problema ou a atingir um objetivo (MITCHELL, 1970). Destaca-se a importância do envolvimento no processamento mental do consumo de alimentos orgânicos (AERTSENS et al., 2009). Consequentemente, incorporar o conceito de envolvimento, considerado como importante elemento moldador de atitudes e intenções comportamentais, possibilita compreender como as motivações do consumidor de orgânicos influenciam o desenvolvimento de determinado comportamento (TENG; LU, 2016). 
Nesse sentido, algumas perguntas emergem e norteiam os interesses deste trabalho: i) como se dá o envolvimento com o produto orgânico? ii) O consumidor possui alto envolvimento com o produto orgânico? iii) Que aspectos podem ser considerados determinantes para o alto envolvimento? e iv) $\mathrm{O}$ consumidor altamente envolvido possui orientações motivacionais egoístas ou altruístas? Portanto, o objetivo deste trabalho é compreender o envolvimento dos consumidores de produtos orgânicos. Além desta introdução, este trabalho traz seções de revisão de literatura sobre envolvimento e produtos orgânicos, procedimentos metodológicos, análise e discussão dos resultados e conclusões.

\section{PRODUTOS ORGÂNICOS E ENVOLVIMENTO}

A revisão de literatura desenvolvida neste trabalho se debruçou sobre o construto multidimensional de envolvimento, incluindo sua mensuração e os determinantes do alto envolvimento com produtos orgânicos.

\subsection{O envolvimento e sua multidimensionalidade}

O envolvimento consiste na relevância percebida de um produto, alicerçada nos valores, necessidades e interesses intrínsecos do indivíduo (ZAICHKOWSKY, 1985), sendo um dos aspectos mais relevantes para moldar o comportamento do consumidor durante o processo decisório (ENGEL; BLACKWELL; MINIARD, 2000). Quester e Lim (2003) acrescentam que o comportamento do consumidor é influenciado pelo nível de envolvimento com o produto e pela situação de consumo antecipada. A literatura oferece escalas para mensurar o envolvimento do consumidor com o produto (ZAICHKOWSKY, 1985; 1994; LAURENT; KAPFERER, 1985; JAIN; SRINIVASAN, 1990), inclusive adaptadas ao contexto brasileiro (FONSECA; ROSSI, 1999; HEPP, 2008; ALMEIDA; DÜSENBERG, 2014). Jain e Srinivasan (1990) desenvolveram análises empíricas com distintas escalas propostas por autores à época para criar e validar a escala New Involvement Profile (NIP).

O construto envolvimento é complexo e tem de ser mensurado numa escala multidimensional (JAIN; SRINIVASAN, 1990). Pesquisas verificaram a existência da multidimensionalidade do envolvimento de consumidores com os alimentos convencionais (JAIN; SRINIVASAN, 1990; FONSECA; ROSSI, 1999; VERBEKE; VACKIER, 2004; 
ALMEIDA; DÜSENBERG, 2014). As dimensões importância, prazer, valor simbólico, importância do risco e probabilidade de risco foram encontradas por meio do uso da escala NIP em pesquisas com alimentos (JAIN; SRINIVASAN, 1990; FONSECA; ROSSI, 1999; ALMEIDA; DÜSENBERG, 2014). Nesse sentido, emerge como hipótese a ser verificada neste trabalho que:

H1: o envolvimento com os produtos orgânicos se dá de maneira multidimensional.

\subsection{Mensuração do envolvimento}

À medida que as pesquisas na área de comportamento do consumidor se desenvolviam nos anos 1980, a conceituação e a mensuração do envolvimento dos consumidores passaram a ser foco de pesquisadores e tiveram considerável aumento (MICHAELIDOU; DIBB, 2006).

Embora tenha sido pouco verificada empiricamente, a compra de alimentos, em geral, é muitas vezes assumida como uma atividade de baixo envolvimento, uma vez que são produtos de aquisição e consumo rotineiros (BEHARRELL; DENISON, 1995). Então, mesmo os indivíduos com uma atitude positiva em relação aos alimentos orgânicos podem não ser compradores regulares (THØGERSEN; OLANDER, 2006). No entanto, Tarkiainen e Sundqvist (2009) ressaltam que não está claro se produtos alimentares orgânicos devem ser incluídos nessa categoria de baixo envolvimento, porque comprá-los muitas vezes se relaciona a um conjunto diferente de motivações, como consciência sobre saúde e ambiental, por exemplo.

Alguns estudos investigaram o envolvimento com categorias de produto próximas aos alimentos orgânicos. Vermeir e Verbeke (2006) analisaram o impacto do envolvimento sobre as atitudes e intenções dos consumidores em relação aos produtos alimentares sustentáveis. Os autores concluíram que o envolvimento tem um impacto positivo significativo na atitude em relação à compra de produtos lácteos sustentáveis, que por sua vez se correlaciona fortemente com a intenção de comprá-los. Conforme já verificaram Bezençon e Blili (2010), o envolvimento ao apelo ético de produtos é muito mais forte do que o envolvimento com as suas características tangíveis, e os consumidores altamente envolvidos preferem canais de venda coerentes com a proposta ética, por exemplo. 
Chen (2007) utilizou a escala Food Involvement Scale (FIS) (BELLA; MARSHALL, 2003) em pesquisa com alimentos orgânicos e concluiu que os consumidores são, geralmente, altamente envolvidos, tendendo os mais envolvidos a ter mais intenção de comprar alimentos orgânicos sem se importarem com a complexidade informacional e de escolha típicas desse produto. Em outras pesquisas com alimentos orgânicos, alguns autores, embora não tenham efetivamente mensurado, supuseram que se trata de um produto de alto envolvimento, dadas as motivações específicas para o consumo e as complexidades informacionais que carregam as tomadas de decisão para a alimentação orgânica (GRUNERT; JUHL, 1995; ZANOLI; NASPETTI, 2002).

O envolvimento influencia resultados comportamentais, tais como: pesquisa de informação, duração do processo de tomada de decisão, comportamento de busca de variedades, comportamento de mudança de marca e compromisso de marca ou lealdade (BEHARRELL; DENNISON, 1995; VERBEKE; VACKIER, 2004; TARKIAINEN; SUNDQVIST, 2009). Dias et al. (2016) criaram e validaram uma escala de lealdade, denominada Scale of Consumer Loyalty to Organic Food (SLOF), e constataram uma grande fidelidade dos consumidores de orgânicos brasileiros. No entanto, o envolvimento do produto e o comprometimento da marca (lealdade) são, na verdade, construções distintas, não necessariamente relacionadas, devendo essas influências serem examinadas independentemente (WARRINGTON; SHIM, 2000).

Nesse sentido, ainda que sejam construtos distintos, o envolvimento é compreendido como elemento predecessor à lealdade (BEHARRELL; DENNISON, 1995; VERBEKE; VACKIER, 2004; TARKIAINEN; SUNDQVIST, 2009). Dessa maneira, se há grande lealdade dos consumidores brasileiros de orgânicos (DIAS et al., 2016) e se se consideram os resultados empíricos quanto às características desse produto (GRUNERT; JUHL, 1995; ZANOLI; NASPETTI, 2002; CHEN, 2007; TARKIAINEN; SUNDQVIST, 2009; BEZENÇON; BLILI, 2010; TENG; LU, 2016), tem-se a hipótese:

H2: os consumidores de produtos orgânicos tendem a possuir alto envolvimento.

\subsection{Determinantes de envolvimento}

Os antecedentes do envolvimento com o produto podem variar para distintas categorias de produtos (MICHAELIDOU; DIBB, 2006). A revisão de literatura permitiu destacar seis 
categorias de análise empiricamente reconhecidas por impactar positiva ou negativamente as atitudes de compra de consumidores. Esses aspectos, cada um apresentado em uma das seis subseções a seguir, foram tratados como variáveis proxy do alto envolvimento, dado que os estudos sobre envolvimento com produtos orgânicos ainda são incipientes.

\subsubsection{Frequência de compra}

Em estudo comparativo entre consumidores regulares e ocasionais, Zanoli e Naspetti (2002) consideraram que a saúde é o motivo mais importante na compra de alimentos orgânicos entre os dois tipos de consumidores. Além disso, para os frequentes, os atributos de saúde estão associados a valores transcendentais de altruísmo e ecologia, enquanto os compradores ocasionais são particularmente atraídos pela satisfação pessoal, isto é, querem que o produto seja saboroso e de boa aparência (ZANOLI; NASPETTI, 2002).

Se os atributos de um produto estiverem relacionados com os motivos de consumo de um indivíduo, o indivíduo será incitado por um certo nível de envolvimento ou excitação emocional, o que levará o indivíduo a perceber o produto como relevante para ele (SCHMIDT; FRIEZE, 1997). Esse envolvimento levaria os consumidores a contemplar a associação do produto com seus valores, necessidades ou benefícios inerentes e estimular ainda mais a ocorrência de comportamentos de compra (TENG; LU, 2016). Os usuários frequentes parecem ter uma estrutura cognitiva mais idealista do que os consumidores ocasionais, e isso pode ser considerado um sintoma de maior envolvimento (ZANOLI; NASPETTI, 2002). Portanto, assume-se neste estudo que:

H3a: a frequência de compra influencia positivamente o alto envolvimento.

\subsubsection{Fonte de informações}

Em relação às fontes de orientação alimentar, estudos mostram que os consumidores ainda são bastante desconfiados e céticos quanto às informações disponibilizadas (MAKATOUNI, 2002). Ainda que os indivíduos associem fortemente a saúde à dieta e, por isso, se interessem por saúde e nutrição (SCHIFFERSTEIN; OPHUIS, 1998; SQUIRES et al., 2001), são bastante críticos com a desinformação de médicos quanto aos orgânicos (MAKATOUNI, 2002). 
De maneira geral, Makatouni (2002) identificou fontes diversas de orientação alimentar: governo (fonte especializada), médicos, nutricionistas e outros profissionais de saúde (orientação profissional), mídia e varejistas (fontes não especializadas). Há também os consumidores determinados a colher por si mesmos informações, especialmente quanto à saúde (SCHIFFERSTEIN; OPHUIS, 1998; SQUIRES et al., 2001). Esses, no entanto, gostariam que existisse mais publicidade nas informações e mais rigor nas certificações, com evidências claras de que órgãos confiáveis atestam a qualidade (MAKATOUNI, 2002). Conforme sugeriram Bravo et al. (2013), o interesse pela busca de informação é um importante preditor do comportamento do consumidor no mercado de alimentos (mídia de massa, mídia específica, especialistas, organizações públicas e familiares), e Schaefer (1997) identificou que indivíduos de maior envolvimento são os mais interessados em se aprofundar sobre informações relacionadas a produtos, razões por que esta pesquisa assume que:

$\mathbf{H}_{3 \mathbf{b}}$ : a busca de informações influencia positivamente o alto envolvimento.

\subsubsection{Aspectos motivadores}

As motivações para o consumo de alimentos orgânicos, conforme Hemmerling et al. (2015) identificaram em grande parte dos estudos, resumem-se em três principais: consciência sobre a saúde, segurança alimentar e proteção ambiental. Há, todavia, diversas outras questões difusas - sejam elas aspectos derivados das três razões principais ou não - mencionadas em outros estudos que contribuiriam para o consumo. De acordo com Hill e Lynchehaun (2002), os consumidores conscientes da saúde percebem os alimentos orgânicos como capazes de melhorar sua saúde, por tenderem a acreditar que são mais nutritivos. A propósito da preocupação com a segurança alimentar, esta dimensão está presente na literatura como uma das fortes razões para o consumo de orgânicos, estando atrelada às questões de naturalidade (livre de agrotóxicos) e de confiança na autenticidade do produto (SCHIFERSTEIN; OPHUIS, 1998; VEGA-ZAMORA et al., 2014).

Como destacam Hoppe, Vieira e Barcellos (2013), no Brasil, além das preocupações habituais com saúde e segurança alimentar, os consumidores também estão preocupados com questões sociais e de proteção ambiental. De acordo com Honkanen et al. (2006), os motivos ecológicos referem-se à preocupação com os direitos ambientais e animais. E, conforme Hughner et al. (2007), as questões sociais envolvem a atenção sobre agricultura familiar e a 
produção local. A constituição física dos produtos, tais como melhor sabor e maior durabilidade, também integra o conjunto de atributos percebidos como relevantes na decisão de compra dos produtos orgânicos (HUGHNER et al., 2007).

Por fim, as normas subjetivas ou sociais para um comportamento ambientalmente responsável estão fundamentadas na literatura (BIEL; THØGERSEN, 2007; THØGERSEN, 2007) para explicar o consumo de orgânicos. De acordo com Biel e Thøgersen (2007), norma subjetiva é a pressão social percebida para uma pessoa se engajar ou não em um comportamento e é determinada pelo conjunto de crenças normativas acessíveis relativas às expectativas de referências importantes para essa pessoa (família ou amigos, por exemplo). No contexto da escolha de alimentos orgânicos, Thøgersen (2007) encontrou uma associação positiva significativa entre a intenção de compra dos consumidores de alimentos orgânicos e sua norma subjetiva. No Brasil, embora existam pesquisadores que não conseguiram verificar a influência das normas sociais (SAMPAIO; GOSLING, 2014; BLANCO; VIEIRA; MIURA, 2018), há pesquisas que evoluíram no sentido de confirmar o papel das normas subjetivas (WATANABE; BRANC; ALFINITO, 2018). Portanto, dada a complexidade de motivações encontradas na literatura, presume-se que:

H3c: os aspectos motivadores impactam positivamente o alto envolvimento.

\subsubsection{Inibidores da compra}

Alguns estudos tratam do preço e outros aspectos como obstáculos que inibem o consumo de alimentos orgânicos. A disponibilidade irregular, ou seja, a quantidade real ou percebida ofertada pelos produtores, bem como a falta de diversidade são inconveniências associadas à compra de alimentos orgânicos (GUIVANT, 2003; CHRYSSOCHOIDIS; KRYSTALLIS, 2005; AERTSENS et al., 2009; BRAVO et al., 2013). Além da disponibilidade irregular, alguns autores relacionam a própria descontinuidade de alguns alimentos como fator negativo sobre a confiança em alimentos orgânicos (ZANOLI; NASPETTI, 2002; GUIVANT, 2003; HUGHNER et al., 2007). A aparência também é geralmente mencionada como um aspecto negativo, tornando-se um impedimento da compra (MAKATOUNI, 2002; ZANOLI; NASPETTI, 2002; AERTSENS et al., 2009). No entanto, a rejeição dos preços elevados é tida na literatura como a principal barreira (MAGNUSSON et al., 2001; HILL; LYNCHEHAUN, 
2002; ZANOLI; NASPETTI, 2002; GUIVANT, 2003; HUGHNER et al., 2007; AERTSENS et al., 2009). Portanto, em relação aos aspectos inibidores, supõe-se que:

H3d: a inibição impacta negativamente o alto envolvimento.

\subsubsection{Disposição ao ágio}

Relativamente à percepção de preço, alguns consumidores acreditam que o alimento orgânico é caro. Isso, porém, segundo pesquisas sobre o perfil de consumidores, não os desencoraja da compra (MAKATOUNI, 2002). De acordo com Aertsens et al. (2009), há consumidores interessados em pagar o preço premium que o produto orgânico possui, por ele ser livre de pesticidas e possuir outros benefícios. Embora, para alguns consumidores, o preço dos alimentos orgânicos seja relativamente alto e os benefícios ainda careçam de evidências (MAKATOUNI, 2002), pesquisas têm observado que, quando os alimentos orgânicos são mais baratos, os consumidores tendem a inferir que são de baixa qualidade e oferecem menos benefícios (HUGHNER et al., 2007). Nesse contexto, supõe-se:

H3e: a disposição ao ágio tem influência positiva sobre o alto envolvimento.

\subsubsection{Aspectos sociodemográficos}

Muitas pesquisas examinaram as características sociodemográficas dos compradores de alimentos orgânicos, mas os resultados se apresentam inconsistentes no Brasil (HOPPE et al., 2012; BLANCO; VIEIRA; MIURA, 2018) e no mundo (BRAVO et al., 2013), seja por adotarem metodologias distintas, seja por terem objetivos díspares ou mesmo porque os perfis apresentam mudanças em função do tempo. Embora os dados demográficos sejam geralmente considerados importantes para prever o consumo de alimentos orgânicos, as comparações entre os estudos mostram discrepâncias em termos de significância (BRAVO et al., 2013), razão pela qual se sugere que:

H3f: as variáveis sociodemográficas não são determinantes de alto envolvimento. 


\section{PROCEDIMENTOS METODOLÓGICOS}

Esta pesquisa classifica-se como descritiva quanto ao objetivo e tem natureza quantitativa (COOPER; SCHINDLER, 2011). Em relação ao procedimento técnico, adotou-se um levantamento do tipo survey. Compreendeu o universo deste estudo o conjunto de consumidores de alimentos orgânicos residentes no Brasil, considerado nesta pesquisa como um conjunto infinito, uma vez que se trata de uma população desconhecida. Para fins de inclusão na amostra, utilizou-se como critério a autodeclaração de consumidor de orgânicos, ainda que não regular. Para a coleta, empregou-se um questionário em plataforma de pesquisa on-line, divulgado em fóruns temáticos, grupos universitários e ciclos sociais dos autores, os quais também promoveram divulgação. Foram coletadas 412 observações válidas, obtendo-se uma representatividade de 22 unidades federativas do país, contemplando todas as regiões geográficas. Um resumo do perfil da amostra é apresentado na Tabela 1.

Tabela 1 - Perfil sociodemográfico da amostra $(\% ; n)$

\begin{tabular}{|c|c|c|c|c|}
\hline \multicolumn{5}{|l|}{$\operatorname{Sexo}(N=409)$} \\
\hline Masculino & Feminino & & & \\
\hline $28,4 \%$ & $71,6 \%$ & & & \\
\hline \multicolumn{5}{|c|}{ Estado civil $(\mathrm{N}=409)$} \\
\hline Solteiro & Casado & $\begin{array}{r}\text { União } \\
\text { Consensual }\end{array}$ & Divorciado & Viúvo \\
\hline $41,8 \%$ & $38,9 \%$ & $12,0 \%$ & $6,4 \%$ & $1,0 \%$ \\
\hline \multicolumn{5}{|c|}{ Escolaridade $(\mathrm{N}=410)$} \\
\hline $\begin{array}{r}\text { Ens. } \\
\text { Fundamental }\end{array}$ & Ens. Médio & Ens. Superior & $\begin{array}{r}\text { Pós-graduação (lato } \\
\text { senso) }\end{array}$ & $\begin{array}{r}\text { Pós-graduação (stricto } \\
\text { sensu) }\end{array}$ \\
\hline $0,0 \%$ & $3,9 \%$ & $30,0 \%$ & $25,4 \%$ & $40,7 \%$ \\
\hline \multicolumn{5}{|c|}{ Idade em anos $(\mathrm{N}=409)$} \\
\hline Média & Moda & Mínimo & Máximo & \\
\hline 36,71 & 36 & 17 & 69 & \\
\hline \multicolumn{5}{|c|}{$\begin{array}{l}\text { Tamanho familiar em número de pessoas } \\
(\mathrm{N}=408)\end{array}$} \\
\hline Média & Moda & Mínimo & Máximo & \\
\hline 2,57 & 2 & 1 & 7 & \\
\hline \multicolumn{5}{|c|}{ Renda mensal familiar $(\mathrm{N}=386)$} \\
\hline Média & Moda & Mínimo & Máximo & \\
\hline $\mathrm{R} \$ 8710,41$ & $\mathrm{R} \$ 10.000,00$ & $\mathrm{R} \$ 500,00$ & $\mathrm{R} \$ 200.000,00$ & \\
\hline
\end{tabular}

Fonte: elaboração própria.

No que tange ao instrumento, visando atender aos objetivos da pesquisa, abordaram-se questões fundamentadas na revisão de literatura sobre: hábitos de compra (frequência de compra geral e por estabelecimento); disposição ao ágio em relação ao alimento convencional; aspectos inibidores da compra; uso e tipos de orientação alimentar; fatores motivadores da predileção por alimentos orgânicos; fatores relacionados ao envolvimento com este tipo de 


\section{ALÉM DA FILOSOFIA ALTRUÍSTA: UM ESTUDO SOBRE OS DETERMINANTES DO ENVOLVIMENTO DO CONSUMIDOR DE ALIMENTOS ORGÂNICOS}

produto; e questões sociodemográficas. O Quadro 1 sintetiza o instrumento com as variáveis e escalas utilizadas.

Quadro 1 - Síntese do instrumento com as variáveis e escalas utilizadas

\begin{tabular}{|c|c|c|}
\hline $\begin{array}{l}\text { Construto / } \\
\text { Variável }\end{array}$ & Questão & Escala \\
\hline \multirow[b]{2}{*}{$\begin{array}{l}\text { Frequência de } \\
\text { compra }\end{array}$} & $\begin{array}{l}\text { Qual a sua frequência de compra de alimentos } \\
\text { orgânicos? } \\
\text { - Não mantenho regularidade; } \\
\text { - Normalmente, ao menos uma vez ao mês; } \\
\text { - Normalmente, ao menos uma vez a cada duas } \\
\text { semanas; } \\
\text { - Normalmente, ao menos uma vez por semana; } \\
\text { - Várias vezes por semana. }\end{array}$ & $\begin{array}{l}\text { Ordinal, variando de "Não } \\
\text { mantenho regularidade" (1) a } \\
\text { "várias vezes por semana" (5) }\end{array}$ \\
\hline & $\begin{array}{l}\text { Qual a sua frequência de compra de } \\
\text { alimentos orgânicos nos estabelecimentos } \\
\text { relacionados? } \\
\text { - Supermercados; } \\
\text { - Feiras Livres; } \\
\text { - Restaurantes Especializados; } \\
\text { - Lojas Especializadas; } \\
\text { - Delivery; } \\
\text { - Comerciantes Ambulantes; e } \\
\text { - Outros. }\end{array}$ & $\begin{array}{l}\text { Ordinal, variando de "Nunca" } \\
\text { (1) a "Sempre" (5) }\end{array}$ \\
\hline $\begin{array}{ll}\text { Fonte } & \mathrm{de} \\
\text { informação } & \end{array}$ & $\begin{array}{l}\text { Como se dá a sua orientação alimentar? } \\
\text { - Não sigo orientações; } \\
\text { - Faço uso de orientações profissionais (médicos, } \\
\text { nutricionistas etc.); } \\
\text { - Sigo orientações de pessoas e/ou grupos que } \\
\text { considero; referências no assunto; } \\
\text { - Costumo consultar fontes especializadas } \\
\text { (livros, revistas científicas etc.); } \\
\text { - Costumo me informar por conta própria, mas } \\
\text { nem sempre recorro a fontes especializadas. }\end{array}$ & $\begin{array}{l}\text { Ordinal do tipo Likert, de cinco } \\
\text { pontos, variando de "discordo } \\
\text { totalmente" (1) a "concordo } \\
\text { totalmente" (5) }\end{array}$ \\
\hline $\begin{array}{l}\text { Motivação para } \\
\text { o consumo }\end{array}$ & $\begin{array}{l}\text { Sobre o alimento orgânico, até que ponto você } \\
\text { concorda ou discorda das seguintes } \\
\text { afirmativas? } \\
\text { - É mais saboroso que o comum; } \\
\text { - É mais saudável que o comum; } \\
\text { - Tem uma durabilidade maior em relação ao } \\
\text { comum; } \\
\text { - Não possui agrotóxicos; } \\
\text { - Não agride o meio ambiente; } \\
\text { - Promove uma função social importante com a } \\
\text { agricultura familiar; } \\
\text { - Privilegia a produção local; } \\
\text { - Costuma ser autenticamente orgânico; e } \\
\text { - É consumido por pessoas que admiro. }\end{array}$ & $\begin{array}{l}\text { Ordinal do tipo Likert, de cinco } \\
\text { pontos, variando de "discordo } \\
\text { totalmente" (1) a "concordo } \\
\text { totalmente" (5) }\end{array}$ \\
\hline
\end{tabular}




\section{(CONTINUAÇÃO)}

\begin{tabular}{|c|c|c|}
\hline $\begin{array}{l}\text { Inibição para o } \\
\text { consumo }\end{array}$ & $\begin{array}{l}\text { Você deixa(ria) de consumir alimento } \\
\text { orgânico porque... } \\
\text { - O preço é normalmente acima se comparado ao } \\
\text { alimento comum; } \\
\text { - A disponibilidade é insuficiente em } \\
\text { estabelecimentos e feiras; } \\
\text { - Há pouca diversidade dos tipos de alimentos; } \\
\text { - Há descontinuidade na venda de alguns } \\
\text { alimentos; } \\
\text { - A aparência não é melhor que a do alimento } \\
\text { comum. }\end{array}$ & $\begin{array}{l}\text { Ordinal do tipo Likert, de cinco } \\
\text { pontos, variando de "discordo } \\
\text { totalmente" (1) a "concordo } \\
\text { totalmente" (5) }\end{array}$ \\
\hline $\begin{array}{l}\text { Disposição ao } \\
\text { ágio }\end{array}$ & $\begin{array}{l}\text { Quanto a mais você se dispõe ou se disporia a } \\
\text { pagar por um alimento orgânico em relação a } \\
\text { um alimento convencional? } \\
\text { - Nada a mais; - Até } 10 \% \text {; - Até } 20 \% \text {; - Até } 30 \% \text {; } \\
\text { - Até } 50 \% \text {; - O dobro ou mais. }\end{array}$ & Ordinal \\
\hline Envolvimento & $\begin{array}{l}\text { Expresse sua opinião sobre o alimento } \\
\text { orgânico: } \\
\text { - Atributos da escala NIP adaptada. (ver Quadro } \\
\text { 2) }\end{array}$ & $\begin{array}{l}\text { Ordinal do tipo diferencial } \\
\text { semântico, de sete pontos }\end{array}$ \\
\hline Sexo & $\begin{array}{l}\text { Qual o seu gênero? } \\
\text { - Masculino; - Feminino. }\end{array}$ & Nominal \\
\hline Idade & Qual a sua idade? & Razão \\
\hline Estado civil & $\begin{array}{l}\text { Qual o seu estado civil ou conjugal? } \\
\text { - Solteiro; - Casado; - União consensual; - } \\
\text { Divorciado; - Viúvo. }\end{array}$ & Nominal \\
\hline $\begin{array}{l}\text { Tamanho } \\
\text { familiar }\end{array}$ & $\begin{array}{l}\text { Contando com você, quantas pessoas residem } \\
\text { em sua casa? }\end{array}$ & Razão \\
\hline $\begin{array}{l}\text { Unidade } \\
\text { federativa }\end{array}$ & $\begin{array}{l}\text { Em qual estado você reside? } \\
\text { - Todos os estados do país, mais o Distrito } \\
\text { Federal. }\end{array}$ & Nominal \\
\hline Escolaridade & $\begin{array}{l}\text { Qual a sua escolaridade? } \\
\text { - Ens. Fundamental (completo ou não); - Ens. } \\
\text { Médio (completo ou não); - Ens. Superior } \\
\text { (completo ou não); - Pós-graduação (lato sensu); } \\
\text { - Pós-graduação (stricto sensu). }\end{array}$ & Nominal \\
\hline Renda & $\begin{array}{l}\text { Em média, quanto você estima que é a sua } \\
\text { renda familiar mensal? }\end{array}$ & Razão \\
\hline
\end{tabular}

Fonte: elaboração própria.

Em relação ao construto envolvimento, o instrumento traz a escala New Involvement Profile (NIP) (JAIN; SRINIVASAN, 1990) traduzida para o português por Fonseca e Rossi (1999). Para este trabalho, dado que o produto pesquisado é bastante específico, optou-se por 


\section{ALÉM DA FILOSOFIA ALTRUÍSTA: UM ESTUDO SOBRE OS DETERMINANTES DO ENVOLVIMENTO DO CONSUMIDOR DE ALIMENTOS ORGÂNICOS}

fazer adaptações na escala, conforme demonstra o Quadro 2. Continuaram dispostos quinze itens relacionados às cinco dimensões, com suas disposições inversas originais (extremos positivos e negativos alternam de posição em alguns atributos da escala). Manteve-se a maior parte dos itens originais, mas fez-se em outros uma adaptação de termos, e alguns itens originais, por fim, foram suprimidos, dada a impertinência em relação ao produto orgânico. Para os casos em que o item original foi suprimido, buscou-se outro que pertencesse à mesma dimensão, privilegiando também os itens de maior carga fatorial na pesquisa.

Quadro 2 - Versão adaptada da escala New Involvement Profile (NIP)

\begin{tabular}{|l|}
\hline Dimensão Relevância \\
\hline 1. Essencial - Não essencial* \\
2. Benéfico - Não benéfico* \\
3. Desnecessário - Necessário \\
\hline
\end{tabular}

Dimensão Prazer

4. Eu não acho prazeroso - Eu acho prazeroso

5. Não é sensorialmente (aparência, sabor, cheiro etc.) agradável - É sensorialmente (aparência, sabor, cheiro etc.) agradável

6. Ao comprá-lo sinto-me satisfeito - Ao comprá-lo não me sinto satisfeito*

\section{Dimensão Valor Simbólico}

7. Fala aos outros sobre mim (é a minha cara) - Não fala aos outros sobre mim (não é a minha cara)*

8. Sofro julgamentos quando não os consumo - Não sofro julgamentos quando não os consumo*

9. Não expressa uma visão dos meus valores para os outros - Expressa uma visão dos meus valores para os outros

\section{Dimensão Importância de Risco}

10. É realmente irritante fazer uma compra inadequada - Não é irritante fazer uma compra inadequada*

11. Uma má escolha não seria um transtorno em termos de qualidade - Uma má escolha seria um transtorno em termos de qualidade

12. Pouco a perder por não escolher um alimento autenticamente orgânico - Muito a perder por não escolher um alimento autenticamente orgânico

\section{Dimensão Probabilidade de Risco}

13. Ao adquiri-lo, estou certo da minha predileção por orgânicos - Ao adquiri-lo, não estou certo da minha predileção por orgânicos

14. Nunca sei se estou de fato comprando um alimento orgânico - Tenho certeza de que estou de fato comprando um alimento orgânico*

15. Eu me sinto um pouco perdido(a) ao escolher isto - Eu não me sinto perdido(a) ao escolher isto*

* representa itens dispostos inversamente

Fonte: adaptado de Fonseca e Rossi (1999). 


\subsection{Operacionalização dos objetivos e hipóteses}

Visando operacionalizar os objetivos, para obter as dimensões de envolvimento, utilizou-se a análise fatorial exploratória, com extração oblíqua, conforme a metodologia original e aplicações posteriores da escala NIP (JAIN; SRINIVASAN, 1990; FONSECA; ROSSI, 2000). Empregou-se como critério o número de fatores com autovalores acima de 1. Retiraram-se, um a cada estágio, dois itens da escala em função de suas comunalidades e cargas fatoriais baixas: no primeiro estágio, a variável "sofro julgamentos pelo não consumo", que apresentou comunalidade insuficiente $(0,40)$ e carga fatorial baixa $(0,364)$; em seguida, a variável "possui meus valores", em função de sua comunalidade inadequada $(0,44)$. Por fim, encontrou-se uma solução com treze itens agrupados a quatro fatores, a qual é apresentada na seção de resultados. Constatou-se que o construto é confiável com treze itens (Alfa de Cronbach $=0,833$ ), bem como se mostrou apropriado à amostra (teste $\mathrm{KMO}=0,868$ ), além do que as variáveis não são correlacionadas nesta população (teste BMT $=1962,879$, com p<0,001). As comunalidades dos treze itens se mostraram adequadas, e a variância total explicada para esta solução foi apropriada ao número de fatores, explicando $66,85 \%$.

Para mensurar o nível de envolvimento, observou-se a média geral das respostas da escala de envolvimento, que - em função de sua formatação de diferencial semântico de sete pontos - resultou em um escore entre 1 e 7, admitindo-se duas casas decimais para fins de melhor análise. Nesse sentido, de maneira análoga ao escore PII (ZAICHKOWSKY, 1994), o nível de envolvimento do consumidor de orgânico foi estabelecido da seguinte forma: baixo envolvimento, para médias entre 1,00 e 2,99; médio envolvimento, no caso das médias entre 3,00 e 5,00; e alto envolvimento, para indivíduos de média entre 5,01 e 7,00.

Esta pesquisa também buscou, por meio de uma regressão logística, compreender os fatores determinantes para um indivíduo estar na faixa de alto envolvimento. Para tanto, promoveu-se um modelo de regressão em que a faixa de envolvimento é a variável de interesse (dependente) em relação a todas as categorias analisadas no estudo (independentes), conforme o Quadro 1. Consideraram-se outliers os indivíduos contidos na faixa de baixo envolvimento, uma vez que representaram apenas 3\% da amostra. Nesse caso, para operacionalização da regressão logística, a variável faixa de envolvimento se tornou dicotômica à medida que os indivíduos de baixo envolvimento foram excluídos, razão por que os indivíduos de envolvimento médio assumiram valor zero e os de envolvimento alto, valor um. 
Segundo Hair Jr. et al. (2006), são premissas para a regressão logística a multicolinearidade, a ausência de observações atípicas e o tamanho da amostra. A verificação de não existência de multicolinearidade se deu, neste trabalho, a partir do teste do Fator de Inflação de Variância (Variance Inflation Factor - VIF). Os outliers e o tamanho da amostra são aspectos que impactam a multicolinearidade, de maneira que a não existência desta indica que o tamanho amostral e os elementos destoantes são aceitáveis. Neste trabalho, os outliers foram retirados por critério de faixa de envolvimento e, especificamente no modelo de regressão utilizado, respeitou-se o tamanho amostral indicado por Hair Jr. et al. (2006), uma vez a proporção superou a razão de 11 observações para cada variável.

Para verificar o ajuste do modelo de regressão, como sugere Dias Filho e Corrar (2009), utilizou-se o teste de Omnibus ( $\mathrm{Chi}^{2}$ do modelo), que rechaçou a hipótese de os coeficientes da equação serem nulos $(\mathrm{p}<0,05)$, e o Teste de Hosmer e Lemeshow, que indicou não haver diferenças significativas entre os resultados preditos e observados ( $p>0,05)$. Analisaram-se, ainda, como medidas de adequação do modelo, os parâmetros de acerto geral e os percentuais de sensibilidade, de especificidade e de capacidade preditiva positiva e negativa. Para estabelecer as variáveis independentes que configuraram os fatores determinantes de alto envolvimento, implementou-se o procedimento stepwise pelo método de backward, isto é, observando a estatística Wald. Fez-se a retirada uma a uma, em 25 estágios, das variáveis não significativas ( $p>0,05)$, interrompendo-se o processo apenas com parâmetros significativos, ou seja, com probabilidades $\mathrm{p}\left(\mathrm{Wald} \mathrm{Chi}^{2}\right)$ inferiores a $5 \%$.

O modelo final é apresentado no capítulo de resultados, cujas variáveis significativas são as características determinantes para um indivíduo ter alto envolvimento. Uma vez verificadas as variáveis determinantes para o alto envolvimento, observaram-se os parâmetros do modelo e suas odds-ratio (razão de chances). A razão de chances é o efeito que uma variação unitária sofrida pela variável independente tende a produzir na probabilidade de ocorrência da variável dependente (DIAS FILHO; CORRAR, 2009). Nesse sentido, o odds-ratio é um fator multiplicador que foi considerado neste trabalho, bem como os sinais dos coeficientes (Beta) relacionados a cada variável indicaram a relação positiva ou negativa, isto é, aumento ou diminuição, respectivamente, da probabilidade de ocorrência da variável dependente.

O Quadro 3 sintetiza a matriz de amarração metodológica deste estudo, com a descrição dos objetivos, a especificação das hipóteses que fomentaram os objetivos, os principais autores utilizados na formação das hipóteses e a exposição das técnicas de análise utilizadas para o alcance dos objetivos. 
Quadro 3 - Matriz de Amarração Metodológica

\begin{tabular}{|c|c|c|c|}
\hline Objetivos & Hipóteses & Autores & Técnicas de Análise \\
\hline $\begin{array}{lr}\text { Identificar } & \text { as } \\
\text { dimensões } & \text { de } \\
\text { envolvimento com } & \text { o } \\
\text { produto orgânico } & \end{array}$ & $\begin{array}{l}\mathbf{H}_{1} \text { : o envolvimento com os } \\
\text { produtos orgânicos se dá de } \\
\text { maneira multidimensional. }\end{array}$ & $\begin{array}{l}\text { JAIN; } \quad \text { SRINIVASAN } \\
(1990) ; \text { FONSECA; ROSSI } \\
(2000) .\end{array}$ & $\begin{array}{l}\text { Análise Fatorial } \\
\text { Exploratória. }\end{array}$ \\
\hline $\begin{array}{l}\text { Mensurar o nível médio } \\
\text { de envolvimento dos } \\
\text { consumidores } \\
\text { orgânicos }\end{array}$ & $\begin{array}{l}\mathbf{H}_{2}: \text { os consumidores de } \\
\text { produtos orgânicos possuem } \\
\text { alto envolvimento. }\end{array}$ & $\begin{array}{l}\text { CHEN (2007); DIAS et al. } \\
\text { (2016); TENG; LU (2016). }\end{array}$ & $\begin{array}{l}\text { Estatística descritiva } \\
\text { com medidas de } \\
\text { tendência central e } \\
\text { dispersão. }\end{array}$ \\
\hline \multirow{6}{*}{$\begin{array}{l}\text { Identificar os } \\
\text { determinantes para o } \\
\text { alto envolvimento com } \\
\text { o produto orgânico }\end{array}$} & $\begin{array}{l}\text { H3a: a frequência de compra } \\
\text { influencia positivamente o } \\
\text { alto envolvimento. }\end{array}$ & $\begin{array}{l}\text { ZANOLI; } \quad \text { NASPETTI } \\
\text { (2002); } \\
\text { TENG; LU (2016). }\end{array}$ & Regressão Logística. \\
\hline & $\begin{array}{l}\text { H}_{3 \mathbf{b}} \text { : a busca de informações } \\
\text { influencia positivamente o } \\
\text { alto envolvimento. }\end{array}$ & $\begin{array}{l}\text { SCHAEFER (1997); } \\
\text { MAKATOUNI (2002); } \\
\text { BRAVO et al. (2013). }\end{array}$ & Regressão Logística. \\
\hline & \begin{tabular}{llr}
$\mathbf{H}_{3 \mathbf{c}}$ : os & \multicolumn{2}{r}{ aspectos } \\
motivadores & impactam \\
positivamente & o & alto \\
envolvimento. & &
\end{tabular} & $\begin{array}{l}\text { HEMMERLING et al. } \\
(2015) ; \quad \text { THØGERSEN } \\
\text { (2007); HOPPE; VIEIRA; } \\
\text { BARCELLOS (2013) }\end{array}$ & Regressão Logística. \\
\hline & $\begin{array}{l}\text { H3d: a inibição impacta } \\
\text { negativamente o alto } \\
\text { envolvimento. }\end{array}$ & $\begin{array}{l}\text { ZANOLI; NASPETTI, } \\
(2002) ; \text { HUGHNER et al. } \\
(2007)\end{array}$ & Regressão Logística. \\
\hline & $\begin{array}{l}\text { Hзe: a disposição ao ágio } \\
\text { tem influência positiva sobre } \\
\text { o alto envolvimento. }\end{array}$ & $\begin{array}{l}\text { MAKATOUNI (2002); } \\
\text { AERTSENS et al. (2009). }\end{array}$ & Regressão Logística. \\
\hline & \begin{tabular}{llr} 
H3f: as & \multicolumn{2}{c}{ variáveis } \\
sociodemográficas não são \\
determinantes de & alto \\
envolvimento. & & \\
\end{tabular} & $\begin{array}{l}\text { HOPPE et al. (2012); } \\
\text { BRAVO et al. (2013). }\end{array}$ & Regressão Logística. \\
\hline
\end{tabular}

Fonte: elaboração própria.

\section{ANÁLISE E DISCUSSÃO DOS RESULTADOS}

\subsection{Dimensões de envolvimento}

Apesar de guardar similaridade com as cinco dimensões da escala original, os resultados sugerem que o envolvimento do consumidor de alimentos orgânicos se dá em quatro dimensões, conforme demonstra a Tabela 2: 1) prazer atribuído ao consumo e relevância de consumir orgânicos; 2) complexidade na escolha do alimento, referente às questões informacionais e técnicas nas decisões de compra; 3) identificação com o produto, que envolve aspectos simbólicos e satisfação com o consumo, além da convicção sobre a predileção por orgânicos; e 
4) importância do risco, relacionada à qualidade e à adequação das compras.

Tabela 2 - Dimensões de envolvimento com o produto orgânico

\begin{tabular}{l|cccc|c}
\hline $\begin{array}{l}\text { Variáveis da escala } \\
\text { NIP }\end{array}$ & $\begin{array}{c}\text { Prazer e } \\
\text { relevância }\end{array}$ & Complexidade & Identificação & $\begin{array}{c}\text { Importância do } \\
\text { risco }\end{array}$ & Comunal. \\
\hline Prazeroso & $\mathbf{0 , 8 5 7}$ & 0,101 & $-0,100$ & $-0,067$ & 0,66 \\
Necessário & $\mathbf{0 , 8 4 4}$ & 0,009 & $-0,067$ & 0,063 & 0,62 \\
Agradável & $\mathbf{0 , 8 2 0}$ & 0,096 & 0,001 & $-0,136$ & 0,71 \\
Benéfico & $\mathbf{0 , 6 7 9}$ & $-0,192$ & 0,306 & 0,023 & 0,71 \\
Importância da & $\mathbf{0 , 6 4 6}$ & 0,096 & 0,095 & 0,259 & 0,67 \\
Autenticidade & $\mathbf{0 , 6 0 8}$ & $-0,055$ & 0,360 & 0,026 & 0,59 \\
Essencial & $-0,070$ & $\mathbf{0 , 8 7 7}$ & 0,057 & $-0,051$ & 0,75 \\
Certeza na compra & 0,204 & $\mathbf{0 , 7 7 6}$ & 0,045 & 0,053 & 0,73 \\
Difícil escolha & 0,380 & 0,259 & $\mathbf{0 , 7 4 5}$ & 0,017 & 0,62 \\
É a minha cara & $-0,213$ & $-0,132$ & $\mathbf{0 , 6 8 4}$ & 0,010 & 0,76 \\
Satisfação & 0,320 & 0,082 & $\mathbf{0 , 4 7 9}$ & 0,148 & 0,64 \\
Certeza da Predileção & $-0,086$ & $-0,050$ & 0,185 & $\mathbf{0 , 8 7 0}$ & 0,65 \\
Irritação com compras & 0,204 & 0,076 & $-0,320$ & $\mathbf{0 , 6 5 0}$ & 0,57 \\
Inadequadas & & & & & \\
Importância da & & & & & \\
Qualidade & &
\end{tabular}

Alfa de Cronbach $=0,833$

Medida Kaiser-Meyer-Olkin de adequação da amostra $=0,868$

Teste de esfericidade de Bartlett $=1962,879 ;$ Significância $=0,000$

Variância total explicada $=66,85 \%$

Fonte: elaboração própria.

Essa condição de multidimensionalidade do envolvimento dos consumidores com o produto orgânico condiz com os pressupostos teóricos encontrados em pesquisas anteriores (JAIN; SRINIVASAN, 1990; FONSECA; ROSSI, 1999; VERBEKE; VACKIER，2004; ALMEIDA; DÜSENBERG, 2014). Nesse sentido, a hipótese 1 foi aceita, pois comprovou-se a existência de múltiplas dimensões de envolvimento. A propósito do nível de envolvimento, conforme apresenta a Tabela 3, os dados sugerem que o consumidor de orgânicos é altamente envolvido, com escore de 5,06. Verifica-se, no entanto, uma posição bastante próxima ao limiar do nível de médio envolvimento. Ainda que o desvio-padrão $(0,93)$ indique uma margem capaz de variar entre o nível médio e o alto de envolvimento, a hipótese 2 foi aceita neste trabalho, tendo em vista que o escore PII (ZAICHKOWSKY, 1994) considera a média exclusivamente.

Tabela 3 - Frequências e escores das faixas de envolvimento com o produto orgânico

\begin{tabular}{lccc}
\hline Faixa de envolvimento & Frequência (n) & Média (1-7) & Desvio-padrão \\
\hline Nível geral de envolvimento $(1,00 \leq \mu \leq 7,00)$ & 412 & 5,06 & 0,93 \\
Baixo envolvimento $(1,00 \leq \mu \leq 2,99)$ & 10 & 2,41 & 0,43 \\
Médio envolvimento $(3,00 \leq \mu \leq 5,00)$ & 165 & 4,31 & 0,47 \\
Alto envolvimento $(5,01 \leq \mu \leq 7,00)$ & 237 & 5,68 & 0,51 \\
\hline
\end{tabular}

Fonte: elaboração própria. 


\subsection{Determinantes do alto envolvimento}

O modelo apresentado na Tabela 4, cujos testes e taxa de acertos $(74,5 \%)$ o revelaram adequado, reflete o que este estudo classificou como determinantes do alto envolvimento com produtos orgânicos. Conforme apresenta a Tabela 4, são fatores que multiplicam as chances de um consumidor estar na faixa de alto envolvimento: "disposição ao ágio" (4,4x); "motivação pela saúde" $(2,04 x)$; "frequência de compras em feiras" (1,51x); "inibição pela disponibilidade insuficiente" (1,47x); "frequência em restaurantes" (1,4x); "motivação pela ausência de agrotóxicos" (1,3x); “motivação pelo sabor" $(1,27 x)$; “motivação pela admiração" $(1,23 x)$; e "frequência geral de compras" (1,23x). Por outro lado, o aspecto "inibição pela descontinuidade" reduz as chances em $72,3 \%$ a cada grau de concordância de um indivíduo.

Tabela 4 - Modelo de regressão logística para determinantes de envolvimento (variável dependente, com envolvimento médio $=0$ e alto=1) de consumidores de produtos orgânicos

\begin{tabular}{lcccc}
\hline Variáveis & Beta & $\begin{array}{c}\text { Odds- } \\
\text { Ratio }\end{array}$ & $\begin{array}{c}\text { Wald Chi } \\
(\mathbf{p})\end{array}$ & VIF \\
\hline Disposição ao ágio (faixas percentuais) & 1,487 & 4,425 & 0,01 & 1,089 \\
Motivação pela saúde & 0,711 & 2,035 & $<0,01$ & 1,457 \\
Frequência de compras em feiras & 0,413 & 1,512 & $<0,01$ & 1,272 \\
Inibição pela disponibilidade insuficiente & 0,384 & 1,468 & $<0,01$ & 1,494 \\
Freq. compras em restaurantes & 0,339 & 1,404 & $<0,01$ & 1,198 \\
especializados & 0,260 & 1,297 & 0,05 & 1,350 \\
Motivação pela ausência de agrotóxicos & 0,242 & 1,273 & 0,03 & 1,338 \\
Motivação pelo sabor & 0,207 & 1,231 & 0,02 & 1,111 \\
Motivação pela admiração a & 0,205 & 1,228 & 0,02 & 1,541 \\
consumidores & $-0,324$ & 0,723 & $<0,01$ & 1,430 \\
Frequência geral de compras & $-9,043$ & 0,000 & $<0,01$ & - \\
Inibição pela descontinuidade & Constante &
\end{tabular}

Teste Omnibus: Chi $^{2}(10)=146,398$ p $<0,001$; Teste Hosmer e Lemeshow: Chi $^{2}(8)=2,106 \mathrm{p}=0,978$; Acertos do modelo $=74,5 \%$; Odds-ratio $=1,146$

Sensibilidade $=80,5 \%$; Especificidade $=67,7 \%$;

Valor preditivo positivo $=74,1 \%$; Valor preditivo negativo $=75,1 \%$

Fonte: elaboração própria.

Dadas as hipóteses levantadas nesta pesquisa, que consideraram o impacto de seis distintos construtos no alto envolvimento de consumidores de orgânicos, consideraram-se aceitas: $\mathrm{H}_{3 \mathrm{a}}$ (dada a influência de frequência geral, em feiras e restaurantes); $\mathrm{H}_{3 c}$ (dados os impactos de aspectos motivadores como saúde, naturalidade, sabor e normas sociais); $\mathrm{H}_{3 \mathrm{e}}$ (em função do impacto da disposição sobre o ágio) e $\mathrm{H}_{3 \mathrm{f}}$ (tendo em vista que as variáveis sociodemográficas não apresentaram efeito sobre o alto envolvimento). Considerou-se 


\section{ALÉM DA FILOSOFIA ALTRUÍSTA: UM ESTUDO SOBRE OS DETERMINANTES DO ENVOLVIMENTO DO CONSUMIDOR DE ALIMENTOS ORGÂNICOS}

parcialmente aceita $\mathrm{H}_{3 \mathrm{~d}}$ (uma vez que inibição pela descontinuidade apresentou efeito redutor de chances, mas a inibição pela insuficiência da oferta teve impacto positivo) e rejeitada $\mathrm{H}_{3 b}$, uma vez que nenhuma fonte de informação se apresentou como determinante para o alto envolvimento.

A motivação para o consumo de alimentos orgânicos não é um conceito pacificado na literatura. Embora alguns autores argumentem que aspectos altruístas sejam as razões proeminentes para determinar o consumo de alimentos orgânicos (HUGHNER et al., 2007; CHEN, 2007; MICHAELIDOU; HASSAN, 2010; BRAVO et al., 2013), publicações internacionais (AERTSENS et al., 2009) e nacionais (VILAS BOAS et al., 2006; ANDRADE; BERTOLDI, 2012) reputam os valores egocêntricos como motivadores mais fortes para a compra de alimentos orgânicos. O entendimento de Aertsens et al. (2009) parece melhor explicar os resultados deste trabalho, sobretudo quando argumentam que necessidades como saúde, relacionada a valores de segurança e hedonismo, desempenham papel mais relevante na determinação de compras de orgânicos.

Estudos também associam a frequência em feiras e ambientes especializados em orgânicos a usuários de valores mais coletivistas, relacionados a questões ecológicas e sociais (GUIVANT, 2003; HOPPE et al., 2012), inclusive associando estes hábitos a padrões de alto envolvimento (ZANOLI; NASPETTI, 2002), enquanto os consumidores ocasionais utilizariam predominantemente o supermercado. Os achados deste estudo, no entanto, sugerem que orientações egoístas como razões de saúde, naturalidade do alimento, sabor e normais sociais, podem estar relacionadas com as motivações de sujeitos frequentes em ambientes especificamente orgânicos. Este comportamento guarda coerência com a pesquisa de Thøgersen (2007), uma vez que as normas subjetivas a que se submetem os consumidores de orgânicos, isto é, os comportamentos reproduzidos intuitivamente são acentuados quando confiam nas propriedades nutritiva e sanitária dos alimentos, e isto resultaria em maior intenção de compra e disposição de pagar preços superiores.

\section{CONSIDERAÇÕES FINAIS}

Este estudo partiu do objetivo principal de investigar o envolvimento do consumidor de produtos orgânicos, mensurando-o e buscando descobrir: a) em que dimensões ele se dá; e b) quais são os aspectos determinantes na faixa de consumidor de maior envolvimento. Concluiu- 
se que, em média, o consumidor de orgânicos está na faixa de alto envolvimento, ainda que próximo ao limiar do médio. Os resultados também evidenciam uma multidimensionalidade na forma de envolvimento destes consumidores, ou seja, tal envolvimento apresenta quatro aspectos latentes: o sentimento de se alimentar de algo necessário e prazeroso simultaneamente; a complexidade cotidiana de se ater às questões informacionais e técnicas nas decisões de compra; o simbolismo e a satisfação de pertencer a este segmento de consumo; e, por fim, a relevância que esse tipo de consumidor atribui à adequação e à boa qualidade da sua alimentação.

A propósito dos aspectos determinantes para o alto envolvimento, os resultados revelaram hábitos de compra e relações motivadoras e inibidoras de consumo. A assiduidade, de maneira geral, bem como a presença em feiras e em restaurantes específicos estão firmemente associadas ao segmento de maior envolvimento. Além destes, o principal fator a evidenciar um consumidor de alto envolvimento é a sua disposição para o ágio, isto é, a situação em que preços majorados em relação ao alimento convencional não eliminam a pretensão de compra. Sendo o preço o principal inconveniente para a intenção de compra de orgânicos (BRAVO et al., 2013), é coerente concluir que os mais dispostos a pagar serão os mais envolvidos.

As motivações para o consumo têm considerável relevância como antecedente de alto envolvimento com o produto orgânico. Dentre esses fatores, os resultados indicam, sobretudo, padrões egoístas do consumidor, uma vez que a salubridade, a naturalidade, o sabor e as normas sociais são as orientações associadas ao individualismo. Este resultado pode estar associado ao fato de que diferentes culturas possuem valores distintos (AERTSENS et al., 2009).

No que tange às questões inibidoras do consumo, identificou-se a disponibilidade insuficiente como determinante para o alto envolvimento, ou seja, quanto mais relevante a indisponibilidade é para um indivíduo, mais aumentam as suas chances de este ser altamente envolvido. Tal resultado é coerente com duas das dimensões do envolvimento tratadas neste trabalho: a complexidade na escolha de produtos e a importância do risco percebido. Nesse sentido, o sujeito altamente envolvido pode não se ater à peculiaridade de que a oferta do produto sofre variações mais severas que a do convencional. A descontinuidade, por outro lado, tem efeito contrário, isto é, se ela é relevante para um indivíduo, diminuem as chances de este estar na faixa de alto envolvimento. Infere-se, portanto, que o segmento de maior envolvimento compreende melhor a descontinuidade que a irregularidade na quantidade ofertada. Sugere-se aprofundar, em estudos futuros, as relações entre essas variáveis com o fenômeno estudado. 
Nesse contexto, o estudo contribui para a literatura ao revelar que: a) na amostra considerada, o envolvimento com orgânicos se dá de maneira multidimensional; e b) as características determinantes que diferenciam os indivíduos altamente envolvidos podem ser associadas a circunstâncias egoístas e a situações de compra específicas, como maior frequência e disponibilidade em pagar preços superiores. Embora o estilo de vida coletivista seja amplamente ponderado dentro da literatura como forte preditor do consumo de orgânicos (GUIVANT, 2003; HOPPE et al., 2012) e comumente relacionado a hábitos de regularidade (ZANOLI; NASPETTI, 2002), este estudo revela que a assiduidade também tende a determinar maior envolvimento, mas as motivações altruístas, todavia, não determinam alto envolvimento.

Em termos gerenciais, este trabalho colabora ao propor um modelo de considerável valor preditivo para analisar consumidores de diferentes faixas de envolvimento com produtos orgânicos, ainda não encontrado na literatura nacional e internacional. Em geral, a segmentação de um mercado em função de níveis de envolvimento é muito útil, uma vez que permite aos profissionais de marketing elaborarem estratégias mais efetivas para cada segmento.

A necessidade de buscar informações e os aspectos sociodemográficos não foram evidenciados como fatores preditivos do alto envolvimento, o que contraria, respectivamente, Schaefer (1997) e Blanco, Vieira e Miura (2018). Sugere-se, portanto, que novos estudos aprofundem a investigação sobre sujeitos de alto envolvimento com o produto orgânico, a fim de promover compreensões mais acuradas a respeito das suas motivações e de seus comportamentos de compra e consumo.

\section{REFERÊNCIAS}

AERTSENS, J.; VERBEKE, W.; MONDELAERS, K.; VAN HUYLENBROECK, G. Personal Determinants of Organic Food Consumption: a review. British Food Journal, v. 111, n. 10, p. 1140-1167, 2009.

ALMEIDA, V. M. C.; DÜSENBERG, N. B. Envolvimento do Consumidor com o Produto: comparação das escalas PII e NIP no contexto brasileiro. Revista Brasileira de Gestão De Negócios, São Paulo, v. 16, n. 50, p. 75-95, jan./mar, 2014.

ANDRADE, L. M. S.; BERTOLDI, M. C. Attitudes and motivations towards organic foods consumption in Belo Horizonte, MG. Braz. J. Food Technol., Campinas, v. 15, 2012. 
BEHARRELL, B.; DENISON, T. J. Involvement in a routine food shopping context. British Food Journal, v. 97, p. 24-29, 1995.

BELLA, R.; MARSHALL, D. W. The construct of food involvement in behavioral research: scale development and validation. Appetite, v. 40, p. 235-244, 2003.

BEZENÇON, V.; BLILI, S. Ethical products and consumer involvement: what's new? European Journal of Marketing, v. 44, n. 9, p. 1305-1321, 2010.

BIEL, A.; THØGERSEN, J. Activation of social norms in social dilemmas: a review of the evidence and reflections on the implications for environmental behaviour. Journal of Economic Psychology, v. 28, p. 93-112, 2007.

BLANCO, T. H. M.; VIEIRA, R. P.; MIURA, M. N. Fatores que Influenciam o Comportamento de Compra de Produtos Orgânicos. In: Seminários em Administração, n. 21, 2018, São Paulo. Anais... São Paulo: SEMEAD, 2018.

BRAVO, C. P.; CORDTS, A.; SCHULZE, B.; SPILLER, A. Assessing determinants of organic food consumption using data from the German National Nutrition Survey II. Food Quality and Preference, v. 28, p. 60-70, 2013.

CHEN, M. F. Consumer attitudes and purchase intentions in relation to organic foods in Taiwan: moderating effects of food-related personality traits. Food Quality and Preference, v. 18, n. 7, p. 1008-1021, 2007.

CHRYSSOCHOIDIS, G. M.; KRYSTALLIS, A. Organic consumers' personal values research: Testing and validating the list of values (LOV) scale and implementing a value-based segmentation task. Food Quality and Preference, v. 16, n. 7, p. 585-599, out. 2005.

COOPER, D. R.; SCHINDLER, P. S. Métodos de pesquisa em administração. Porto Alegre: Bookman, 2011.

DALCIN, D; SOUZA, A. R. L. de; FREITAS, J. B. de; PADULA, A. D.; DEWES, H. Organic products in Brazil: from an ideological orientation to a market choice. British Food Journal, v. 116, n. 12, p. 1998-2015, 2014.

DIAS, V. da V.; SCHUSTER, M. da S.; TALAMINI, E.; RÉVILLION, J. P. Scale of consumer loyalty for organic food. British Food Journal, v. 118, n. 3, p. 697-713, 2016. 
DIAS FILHO, J. M.; CORRAR, L. J. Regressão Logística. In: CORRAR, L. J.; PAULO, E.; DIAS FILHO, J. M. (Coords.). Análise Multivariada: para os cursos de administração, ciências contábeis e economia. São Paulo: Atlas, 2009.

ENGEL, J. F.; BLACKWELL, R. D.; MINIARD, P. W. Comportamento do consumidor. Rio de Janeiro: JC Editora, 2000.

FONSECA, M. J.; ROSSI, C. A. V. Avaliação da aplicabilidade da escala New Involvement Profile para mensuração do envolvimento do consumidor na cidade de Porto Alegre. In: Encontro Nacional da Associação de Pesquisa e Pós-Graduação, n. 23, 1999, Rio Janeiro. Anais... Foz do Iguaçu: ANPAD, 1999.

;___ Involvement research in an international context: evaluation of a scale in Brazil. In: American Marketing Association 2000 Summer Marketing Educators' Conference, 2000, Chicago. Proceedings... Chicago: AMA, 2000. p. 287-296.

GUIVANT, J. S. Os Supermercados na Oferta de Alimentos Orgânicos: apelando ao estilo de vida ego-trip. Ambiente e Sociedade. São Paulo, v. 6, n. 2, jul./dez., p. 63-81, 2003.

GRUNERT, S. C.; JUHL, H. J. Values, environmental attitudes, and buying of organic foods. Journal of Economic Psychology, v. 16, p. 39-62, 1995.

HAIR JR, J. F.; BLACK, W. C; BABIN, B. J; ANDERSON, R. E; TATHAM, R. L. Multivariate Data Analysis. Upper Saddle River, NJ: Pearson Prentice Hall, 2006.

HEMMERLING, S.; HAMM, U.; SPILLER, A. Consumption behaviour regarding organic food from a marketing perspective-a literature review. Organic Agriculture, v. 5, n. 4, p. 277-313, 2015.

HEPP, C P. T. Satisfação, Lealdade e Envolvimento do Consumidor: um estudo no varejo de vestuário. In: Encontro Nacional da Associação de Pesquisa e Pós-Graduação, n. 32, 2008, Rio Janeiro. Anais... Rio de Janeiro: ANPAD, 2008.

HILL, H; LYNCHEHAUN, F. Organic milk: attitudes and consumption patterns. British Food Journal, v. 104, n. 7, p. 526-542, 2002.

HONKANEN, P.; VERPLANKEN, B.; OLSEN, S. O. Ethical values and motives driving organic food choice. Journal of Consumer Behaviour, v. 5, p. 420-430, 2006. 
HOPPE, A.; BARCELlOS, M. D. de; VIEIRA, L. M.; de MATOS, C. A. Comportamento do Consumidor de Produtos Orgânicos: Uma aplicação da Teoria do Comportamento Planejado. Revista Base da UNISINOS, v. 9, n. 2, p. 174-188, 2012.

; VIEIRA, L. M.; BARCELLOS, M. D. de. Consumer behaviour towards organic food in Porto Alegre: an application of the Theory of Planned Behaviour. Rev. Econ. Sociol. Rural, Brasília, v. 51, n. 1, p. 69-90, Mar. 2013.

HUGHNER, R. S.; MCDONAGH, P.; PROTHERO, A.; SHULTZ, C. J.; II; STANTON, J. Who are organic food consumers? A compilation and review of why people purchase organic food. Journal of Consumer Behaviour, v. 6, p. 94-110, 2007.

JAIN, K.; SRINIVASAN, N. An empirical assessment of multiple operationalizations of involvement. Advances in Consumer Research, v. 17, n. 1, p. 594-602, 1990.

LAURENT, G., KAPFERER, J. N. Measuring Consumer Involvement Profiles. Journal of Marketing Research, v. 22, p. 41-53, 1985.

MAGNUSSON, M. K., ARVOLA, A; HURSTI, U; ABERG, L; SJODEN, P. Attitudes towards organic foods among Swedish consumers. British Food Journal, v. 103, n. 3, 2001.

MAKATOUNI, A. What motivates consumers to buy organic food in the UK?: Results from a qualitative study. British Food Journal, v. 104, n. 3/4/5, p. 345-352, 2002.

MICHAELIDOU, N.; DIBB, S. Product Involvement: An Application in Clothing. Journal of Consumer Behavior, v. 5, n. 5, p. 442-453, 2006.

; HASSAN, L. M. Modeling the factors affecting rural consumers' purchase of organic and free-range produce: a case study of consumers from the Island of Arran in Scotland, UK. Food Policy, v. 35, n. 2, p. 130-139, 2010.

MITCHELL, A. Involvement: a potentially important mediator of consumer behavior, in WILKIE, W. L, ed., Advances in Consumer Research. Provo, UT: Association for Consumer Research, p. 191-196, 1970.

ORMOND, J. G. P.; PAULA, S. R. L. de; FAVERET FILHO, P.; ROCHA, L. T. M. Agricultura orgânica: quando o passado é futuro. BNDES, Rio de Janeiro, n. 15, 2002. 
QUESTER, P; LIM, A. L. Product involvement/brand loyalty: is there a link? Journal of Product and Brand Management, Bradford, v. 12, n. 1, p. 22-38, 2003.

SAMPAIO, D.; GOSLING, M. Intenção de compra e consumo de alimentos orgânicos.

Revista Gestão Organizacional, v. 7, n. 1, p. 43-62, 2014.

SCHAEFER, A. Consumer knowledge and country of origin effects. European Journal of Marketing, Bradford, v. 31, n. 1, p. 56-72, 1997.

SCHIFFERSTEIN, H. N. J.; OPHUIS, P. A. M. O. Health-related determinants of organic food consumption in the Netherlands. Food Quality and Preference, v. 9, n. 3, p. 119-133, 1998.

SCHMIDT, L. C.; FRIEZE, I. H. A mediational model of power, affiliation and achievement motives and product involvement. Journal of Business and Psychology, v. 11, n. 4, p. 425446, 1997.

SQUIRES, L; JURIC, B; CORNWELL, T. B. Level of market development and intensity of organic food consumption: cross-cultural study of Danish and New Zealand consumers. Journal of Consumer Marketing, v. 18, n. 5, p. 392-409, 2001.

TARKIAINEN, A; SUNDQVIST, S. Product Involvement in Organic Food Consumption: Does Ideology Meet Practice? Psychology \& Marketing, v. 26, n. 9, p. 844-863, set. 2009.

TENG, C.; LU, C. Organic food consumption in Taiwan: Motives, involvement, and purchase intention under the moderating role of uncertainty. Appetite, v. 105, p. 95-105, out, 2016.

THØGERSEN, J. The motivational roots of norms for environmentally responsible behavior. in Nordic Consumer Policy Research Conference, Helsinki, 2007.

; OLANDER, F. The dynamic interaction of personal norms and environment-friendly buying behavior: a panel study 1. Journal of Applied Social Psychology, v. 36, n. 7, p. 17581780, 2006.

VEGA-ZAMORA, M; TORRES-RUIZ, F. J; MURGADO-ARMENTEROS, E. M.; PARRASROSA, M. Organic as a Heuristic Cue: What Spanish Consumers Mean by Organic Foods. Psychology and Marketing, v. 31, n. 5, p. 349-359, May 2014.

VERBEKE, W.; VACKIER, I. Profile and effects of consumer involvement in fresh meat. Meat Science, v. 67, n. 1, p. 159-168, 2004. 
VERMEIR, I.; VERBEKE, W. Sustainable food consumption: exploring the consumer 'attitude-behavioural intention' gap. Journal of Agricultural and Environmental Ethics, v. 19, n. 2, 2006.

VILAS BOAS, L. H. de B.; SETTE, R. de S.; BRITO, M. J. de. Comportamento do consumidor de produtos orgânicos: uma aplicação da teoria da cadeia de meios e fins. Organizações Rurais \& Agroindustriais, v. 8, n. 1, p. 35-39, 2006

WARRINGTON, P.; SHIM, S. An empirical investigation of the relationship between product involvement and brand commitment. Psychology \& Marketing, v. 17, p. 761-782, 2000.

WATANABE, E. A. M.; BRANC, T. V. C.; ALFINITO, S. Preditores do Consumo de Alimentos Orgânicos: Uma Aplicação da Teoria do Comportamento Planejado. In: Encontro de Marketing da Associação de Pesquisa e Pós-Graduação, n. 8, 2018, Rio Janeiro. Anais... Porto Alegre: EMA, 2018.

ZAICHKOWSKY, J. L. Measuring the involvement construct. Journal of Consumer Research. Bradford, v. 12, n. 3, p. 341-352, Dec. 1985.

. The personal involvement inventory: reduction, revision, and application to advertising. Journal of Advertising, Clemson, v. 23, n. 4, p. 59-70, Dec. 1994.

ZANOLI, R.; NASPETTI, S. Consumer motivations in the purchase of organic food. British Food Journal, v. 104, p. 643-653, 2002. 